\title{
Hipofosfemia en recién nacidos prematuros: un trastorno bimodal
}

\author{
Hypophosphatemia in preterm infants: a bimodal disorder
}

\author{
María Paz Cubillos Celis ${ }^{\mathrm{a}}$, Patricia Mena Nannig ${ }^{\mathrm{b}}$
}

aNeonatóloga, Complejo Asistencial Dr. Sótero del Río

bNeonatóloga, Complejo Asistencial Dr. Sótero del Río, Profesor Titular Adjunto, Pontificia Universidad Católica de Chile.

Recibido el 8 de agosto de 2017; aceptado el 27 de noviembre de 2017

\section{Resumen}

Las estrategias nutricionales para prematuros extremos con alto aporte de proteínas, han mostrado alteraciones metabólicas con hipofosfemia precoz, especialmente en el grupo de pacientes con restricción de crecimiento intrauterino (RCIU). También se presenta hipofosfemia tardía, característica de la enfermedad metabólica ósea. En este artículo se revisan y actualizan conceptos en relación a la fisiopatología del metabolismo del fósforo en recién nacidos prematuros y uso de parenterales precoces en el contexto de enfermedad metabólica ósea. Los artículos fueron identificados en base de datos electrónicas como Pubmed y Rima. Fueron incluidos artículos en inglés y español. Fueron filtrados por título y resumen. La literatura actual propone diversas estrategias de nutrición precoz que permitan asegurar una adecuada cantidad de nutrientes para continuar con el crecimiento y desarrollo extrauterino. En pacientes con nutrición parenteral pero con diferentes aportes de fósforo, o relación calcio:fósforo inadecuada, a mayor contenido de aminoácidos, se presenta hipofosfemia, hipercalcemia, hipomagnesemia, hipokalemia e hiperglicemia, especialmente en casos de RCIU. Estas alteraciones se asocian a prolongación de ventilación mecánica, mayor riesgo de displasia broncopulmonar y aumento de sepsis tardía. La hipofosfemia tardía, descrita ya hace muchos años, se presenta con normocalcemia y aumento de fosfatasas alcalinas, en la enfermedad metabólica ósea del prematuro, con alteración de la mineralización en distintos grados, secundaria a un inadecuado aporte de este mineral para los altos requerimientos de estos pacientes. Esta presentación de hipofosfemia precoz y tardía en el prematuro alerta sobre el control oportuno de fosfemia para ajustar el aporte nutricional. En el prematuro con nutrición parenteral precoz, el control en conjunto con la calcemia en la primera semana de vida, especialmente en RCIU, permite tratar la hipofosfemia y prevenir sus complicaciones. En hipofosfemia tardía, el seguimiento semanal o quincenal desde las 4 semanas a los prematuros con riesgo, permite lograr un aporte óptimo de minerales.
Palabras clave:

Muy bajo peso de nacimiento, prematuro, hipofosfemia, hipercalcemia, enfermedad metabólica ósea, nutrición agresiva 


\begin{abstract}
New nutritional approaches to treat extreme premature babies have demonstrated relevant evidence of metabolic disturbances with early hypophosphatemia, especially in patients with intrauterine growth restriction (IUGR). They have shown late hypophosphatemia, as well, which is characteristic in the metabolic bone disease. A sytematic search of literature describing metabolic disturbances of phosphorus in preterm newborns is presented, related to the use of early parenteral nutrition and also in the context of metabolic bone disease. The articles were gathered from electronic data bases, such as PubMed and Rima. We include articles in english and spanish which were selected by titles and abstracts. Several strategies for early nutrition have been proposed in order to ensure an adequate amount of nutrients to accomplish the development and growth of preterm babies. Patients with parenteral nutrition support with different doses of phosphate, or inadequate calcium phosphate relation, or an increased amino acid content, may present hypophosphatemia, hypercalcemia, hypomagnesemia, hypokalemia and hyperglycemia, all of these are additionally noteworthy in the presence of intrauterine growth restriction. Furthermore, said alterations are associated with prolonged mechanical ventilation, as well as bronchopulmonary dysplasia and increase in late onset sepsis. The late hypophosphatemia, described several years ago, arises as normocalcemia and as an increment of alkaline phosphatases in the metabolic bone disease in preterm babies, and also with an inadequate mineralization in different grades, secondary to an inadequate supply due to high nutritional requirements in these patients. When early or late hypophosphatemia appears in preterm babies, it shall require timely control of phosphemia and will need to adjust the nutritional intake in order to correct it. In case of preterm babies with early parenteral nutrition it will also need a control of calcemia in the first week of birth, especially if those belonging to the IUGR group. Adjustment must be made along with metabolic follow up, as well. In late hypophosphatemia, a weekly or every two weeks follow up will be a must for all preterm babies in risk and they should be given supplements to get an optimum mineral supply.
\end{abstract}

\section{Keywords:}

Very low birth weight infant,

Premature,

Hypophosphatemia, Hypercalcemia, Metabolic Bone Disease, Aggressive Nutrition

\section{Introducción}

El manejo nutricional en pacientes de muy bajo peso de nacimiento (MBPN), representa un desafío constante en las unidades de cuidados intensivos neonatales, en especial los con extremo bajo peso o con restricción del crecimiento intrauterino (RCIU). Las nuevas prácticas clínicas de asistencia nurticional, que aportan precozmente mayor contenido de proteínas, han sido asociadas a alteraciones metabólicas no previstas, como la presentación de hipofosfemia precoz. La descripción de este cuadro tuvo una latencia importante derivada de la práctica de no controlar fosfemia en la primera semana de vida. Más conocida ha sido la hipofosfemia tardía de la enfermedad metabólica ósea del prematuro. Se presenta una actualización de la fisiología, fisiopatología, clínica, prevención y manejo de estos dos trastornos nutricionales del fósforo en el prematuro extremo.

\section{Metabolismo de calcio y fósforo}

El fósforo es el anión intracelular más importante en el organismo, es un componente principal de los huesos y se encuentra involucrado en diversos procesos metabólicos, como producción energética a través de ATP, regulación enzimática y como componente de membranas celulares y ADN. Durante la gestación la acumulación de fósforo no es constante, el $80 \%$ se produce en el tercer trimestre del embarazo, depositando $40 \mathrm{mg} /$ día en la semana 24 de gestación y $75 \mathrm{mg} / \mathrm{kg}$ durante las últimas semanas de gestación ${ }^{1}$.

El proceso de transporte del fósforo a nivel placentario ocurre por transporte activo, transcelular, dependiente de sodio, existiendo un flujo bidireccional, con una alta asimetría en dirección materno-fetal. En condiciones de anoxia, se reduce el flujo de este anión a nivel placentario, como también otros nutrientes. Los factores que modulan este mecanismo de transporte no han sido bien estudiados, sin embargo la presencia de receptores de 1,25 hidroxivitamina $\mathrm{D}$ y paratohormona (PTH) en la placenta, sugieren que podrían estar involucradas. La vitamina $\mathrm{D}$, que es hidrolizada por la placenta, sería un estimulante del transporte de fósforo, aumentando las concentraciones séricas y la PTH fetal actuaría inhibiendo esta transferencia a nivel placentario fetal. La distribución de fósforo es predominantemente en el hueso, el $85 \%$ se encuentra en forma de sales de hidroxiapatita, el porcentaje restante se encuentra en los tejidos blandos (ésteres) formando las membranas fosfolipídicas, ácidos nucleicos, ATP, enzimas y alrededor de un $1 \%$ se encuentra en el líquido extracelular (inorgánicos). Este anión se 
encuentra de dos formas en los fluidos: en una fracción orgánica, compuesta de fosfolípidos y ésteres, y una fracción inorgánica, que se encuentra en plasma y es ultrafiltrable o difusible. Es importante mantener una concentración sérica de fósforo que facilite una buena mineralización ósea y una excreción tubular de fosfato adecuada. Recientemente se ha descrito la participación de fosfatoninas en la regulación de los niveles de fósforo. Las fosfatoninas han permitido entender gran parte de las alteraciones con hipo e hiperfosfemia de causa genética, tumoral y secundarias a insuficiencia renal crónica ${ }^{2}$. Entre las fosfatoninas, se ha descrito el factor de crecimiento de los fibroblastos (FGF23) que aumenta 4 veces entre el nacimiento y los 4-5 días ${ }^{3}$.

El calcio, que tiene una interdependencia con el fósforo, es el mineral más abundante en el organismo y el principal constituyente inorgánico del hueso. Al igual que el fósforo, se acumula preferentemente en el tercer trimestre, en un $80 \%$ del total y se depositan 60 mg/día en la semana 24 de gestación y $150 \mathrm{mg} / \mathrm{k} /$ día en los últimos meses. El 99\% se encuentra en el hueso en forma de hidroxiapatita y el $1 \%$ restante en fluidos extracelulares y tejidos blandos. Se presenta como calcio iónico $(50 \%)$, unido a proteínas $(40 \%)$ y una pequeña parte como complejo difusible (citrato, fosfatos, bicarbonato); las formas no unidas a proteínas difunden las membranas semipermeables. Al nacer, para mantener la homeostasis, se aumenta el flujo de calcio a un 16$20 \%$ desde el hueso al extracelular, mientras se logra un aporte exógeno suficiente. En recién nacidos, especialmente en prematuros ocurre un nadir a las 24- 36 horas de vida, asociado a una falta de respuesta de la glándula paratiroidea secundario a la prematuridad. La PTH en condiciones normales se produce por las bajas concentraciones de calcio y sus funciones son: en el hueso: estimular la resorción para liberar calcio y fósforo al espacio extracelular; en el riñón: aumentar la excreción urinaria de fósforo y disminuir la de calcio; y a nivel gastrointestinal: aumenta indirectamente la absorción de estos minerales a través de la síntesis de 1,25 hidroxivitamina D. La 1,25 hidroxivitamina D, forma activa de la vitamina $\mathrm{D}$, aumenta la absorción intestinal de calcio y fósforo, moviliza ambos para favorecer la mineralización ósea y aumenta la reabsorción de calcio en el riñón ${ }^{4}$.

La calcitonina se produce a nivel placentario y en tiroides fetal y circula a niveles mayores que la madre. $\mathrm{Al}$ nacer, aumenta en las primeras 48 horas y baja de forma gradual posteriormente . $^{5}$

La absorción intestinal en RNPT se ha estimado para el calcio en $50-80 \%$ y en $80 \%$ para el fósforo. El calcio se absorbe en el intestino delgado principalmente en porciones proximales, por medio de dos procesos, el transcelular o activo, predominante en duodeno y yeyuno, y el paracelular o pasivo, a través de uniones estrechas, presente en todo el intestino. Afectan la absorción intestinal de calcio: la presencia de lactosa, el tipo de proteína, el tipo de triglicérido, las cantidades de sodio, zinc, hierro y es mayor desde la leche materna que de las fórmulas ${ }^{5}$. El transporte transcelular ocurre en tres pasos: ingreso por la membrana apical del enterocito a través de canales selectivos de calcio, luego el transporte del calcio intracelular facilitado por la vitamina $\mathrm{D}$ y finalmente se transfiere el ión calcio a una bomba de Ca-ATP asa, que lo libera a la circulación ${ }^{4}$.

En comparación con el calcio, se sabe mucho menos de la absorción intestinal del fósforo. La absorción es mayor, cercana a un $80-90 \%$, y ocurre en todo el intestino delgado. El transporte ocurre también por mecanismos paracelular y transcelular, y este último es dependiente de sodio. El ingreso se produce en el borde en cepillo epitelial, que implica trasporte de sodio, luego cruza el citoplasma a través de mecanismos desconocidos y en la membrana basolateral se mueve por difusión simple o por un mecanismo facilitador. El fósforo tiene varias funciones metabólicas vitales, por lo cual, ante su descenso, se libera a la circulación desde el hueso junto con calcio, por los mecanismos hormonales descritos, produciéndose hipercalcemia e hipercalciuria ${ }^{6}$.

Calcio y fósforo forman parte de la estructura de los huesos, siendo los minerales más abundantes, en forma de hidroxiapatita y carbonato cálcico, en una relación de 2:1 y en menores cantidades se encuentran hidróxido de magnesio, cloruro y sulfato magnésico.

La diferente absorción de calcio y fósforo a nivel intestinal y su proporción a nivel óseo es determinante de las recomendaciones de aporte enteral, mucho mayores para el calcio (120-200 mg/k/día) que para fósforo (60-140 mg K día) $)^{5}$. La proporción de calcio y fósforo a nivel de nutrición parenteral considera superada la barrera intestinal, por lo que se recomienda entre 1 y $1,5: 1$ en $\mathrm{mg} / \mathrm{dl}^{5}$.

La vitamina $\mathrm{D}$ que participa en la absorción intestinal de estos minerales, tiene niveles séricos en prematuros comparables a lactantes sanos (13-63 pg/ml) y varía de acuerdo al estado materno, que es influido por el grupo racial, la exposición solar, la ingesta alimenticia de la madre, la paridad y la estación del año, siendo mayores los niveles en verano que en invierno ${ }^{4,5}$. La recomendación de vitamina $\mathrm{D}$ oral en el prematuro oscila entre 400 y 1,000 UI/día en función de su estado de vitamina $\mathrm{D}^{5}$.

\section{Hipofosfemia precoz}

Se define hipofosfemia como niveles plasmáticos menores de $4,5 \mathrm{mg} / \mathrm{dl}$ o $1,45 \mathrm{mmol} / \mathrm{L}$, moderada entre $2,5 \mathrm{mg} / \mathrm{dl}$ y $1 \mathrm{mg} / \mathrm{dl}(0,8-0,3 \mathrm{mmol} / \mathrm{L})$ y severa si 
el fósforo es menor de $1 \mathrm{mg} / \mathrm{dl}(<0,3 \mathrm{mmol} / \mathrm{L})^{22}$. En el caso de los MBPN, al nacer se interrumpe el paso de nutrientes y la acumulación de los minerales que es fundamental en el tercer trimestre. Hace más de 10 años, la nutrición parenteral se iniciaba tardíamente por el efecto tóxico de los hidrolizados de proteínas de las primeras soluciones parenterales. Con los aminoácidos (Aá) cristalinos se continuó esta práctica hasta los estudios de Thureen que mostraron buen balance nitrogenado y tolerancia metabólica, con el inicio el primer día ${ }^{7}$. Las prácticas clínicas cambiaron gradualmente, con inicio precoz y en niveles más altos, incluso 3 a 3,5 g/k/día desde el primer día, para inhibir el catabolismo e inducir el anabolismo celular. En el estado catabólico, se pierde alrededor de un 1\% diario de las reservas de aminoácidos y de nutrientes intracelulares ${ }^{8}$. Esta pérdida de peso afecta significativamente el crecimiento posterior y la incidencia de la restricción de crecimiento extrauterina (RCEU) determinada por la morbilidad y el insuficiente soporte nutricional. Evitar esta desnutrición permite asegurar un mejor crecimiento y evolución del neurodesarrollo9. Reportes de intervenciones aleatorizadas o históricas mostraron ventajas y buena tolerancia metabólica sin mayores efectos adversos hasta el estudio controlado, aleatorizado de Moltu, que fue suspendido por la presentación de $63 \%$ de sepsis en el grupo con alto y precoz Aá, versus $29 \%$ en el grupo con menor aporte ${ }^{10}$. El aporte de fósforo en este estudio no aumentó con el aporte alto de aminoácidos y la sepsis se observó predominantemente en el grupo hipofosfémico ${ }^{10}$. Bosante revisa datos de estudios de la década previa describiendo el claro aumento de hipofosfemia e hipercalcemia en relación a aumento de Aá.

El incremento de aporte de proteínas se realizó con prácticas variadas respecto a otros nutrientes: inicio precoz de calcio, pero no de fósforo ${ }^{6,11,12}$, relaciones inadecuadas de calcio y fósforo en la parenteral ${ }^{13,14,15}$, o cantidades bajas de fósforo ${ }^{11,16}$. Como consecuencia se observa la presentación frecuente, aunque en muy variable incidencia de hipofosfemia, e hipercalcemia en recién nacidos prematuros extremos, con frecuencia acompañado de hipokalemia, y ocasionalmente hipomagnesemia ${ }^{6,17}$ y mayor aún en los prematuros con $\operatorname{RCIU}^{11,17,18,20}$.

\section{Síndrome de realimentación en prematuros}

La asociación de hipokalemia, hipofosfemia e hipercalcemia, constituirían una modalidad de síndrome de realimentación, que se observaen pacientes prematuros al interrumpir el flujo placentario, especialmente en aquellos con antecedentes de desnutrición intrauterina, con el inicio de nutrición parenteral temprana y agresiva ${ }^{6,19,21,22}$. Estas alteraciones son el reflejo del anabolismo, con aporte parenteral alto en proteínas sin concomitante ajuste de minerales. El principal marcador bioquímico es la hipofosfemia severa y se observa al $3^{\circ}-4^{\circ}$ día de iniciados los nutrientes, pero en severa desnutrición intrauterina puede ser más precoz, primer a segundo día. Además con los carbohidratos y Aá, se produce aumento en los niveles de insulina lo que a su vez estimula la entrada de fósforo y potasio al intracelular, disminuyendo las concentraciones plasmáticas de estos electrolitos. Por otro lado, el anabolismo aumenta las demandas celulares de fósforo, glucosa, potasio y agua.

Desde el punto de vista clínico la hipofosfemia produce dos complicaciones importantes en este período: las infecciones tardías y la prolongación de problemas respiratorios. El aumento de infecciones había sido reportado asociado a nutrición agresiva con controles históricos y fue documentado en un estudio controlado de nutrición agresiva, que fue suspendido por el aumento de la sepsis tardía ${ }^{10}$. En este estudio la hipofosfemia severa $(<2,7 \mathrm{mg} / \mathrm{dl})$ se presentó en el $43 \%$ de los casos y de éstos el 73\% tuvo septicemia comparados con el $37 \%$ de aquellos con hipofosfemia no severa $(<4,2 \mathrm{mg} / \mathrm{dl})$. La sepsis fue corroborada con cultivos positivos. La afectación de la serie blanca, con disminución de la actividad quimiotáctica, fagocítica y bactericida de los granulocitos, ha sido descrita secundaria a la depleción de fosfatos. Los microfilamentos requieren ATP para formar pseudópodos y vacuolas ${ }^{10}$.

La prolongación de problemas respiratorios se produce por dificultad en el retiro del ventilador, mayor duración de requerimientos de oxígeno y de displasia broncopulmonar, producida por la hipotonía durante la hipofosfemia. La hipotonía en hipofosfemia se produce por la depleción de fosfocreatina y ATP a nivel muscular, lo que se ha documentado con la espectroscopía de $\mathrm{RNM}^{24}$ y es potenciada por la hipercalcemia concomitante. En el caso de hipofosfemia severa puede producir falla respiratoria, falla miocárdica por depleción de ATP del miocito, rabdomiólisis, anemia por aumento de la rigidez de los eritrocitos que resulta en hemólisis, convulsiones, acidosis metabólica ${ }^{21,23}$, pero estas últimas no han sido reportadas en el período neonatal actual. También se ha asociado hiperglicemia, y una hipótesis es que esta alteración metabólica precedería a la hiperglicemia a través de dos mecanismos: la hipofosfemia conduciría a un déficit de ATP intracelular que afectaría a las células beta del páncreas, que reduciría la producción de insulina y por otro lado estaría afectada la fosforilación del receptor de insulina, lo cual disminuiría su efectividad ${ }^{25}$. De acuerdo a estos resultados el riesgo de hiperglicemia persistente (>150 mg/dl) aumenta en 3 veces con una disminución de $1,2 \mathrm{mg} / \mathrm{dl}$ de la fosfemia ${ }^{25}$. 
En la tabla 1 se resumen publicaciones sobre la hipofosfemia precoz en el prematuro, con variada incidencia, en parte por las definiciones de valores bajos descritas, pero también por diferente manejo nutricional y grupos estudiados. Del análisis de estas publicaciones se observa que el ser pequeño para la edad gestacional (PEG), a mayores aportes de aminoácidos, el desbalance en relación Ca:P y los aportes insuficientes de fósforo interactúan para determinar hipofosfemia.

Las publicaciones muestran que el fósforo se debe administrar desde el inicio de la nutrición parenteral, con mayor cantidad si se inician aminoácidos en altas dosis. La cantidad de fósforo requerida en la parenteral se puede estimar aproximadamente en base al aporte de calcio y proteínas como $=$ Calcio en $\mathrm{mg} / \mathrm{k} /$ día $/ 2.15+$ (aminoácidos en $\mathrm{g} / \mathrm{K} / \mathrm{d}-1,3)^{\star} 0,8{ }^{\star} 12,3$. Este cálculo no considera el aporte enteral, ni el fósforo necesario para reponer el déficit, pero permite una estimación basal a considerar $^{6,26}$. La relación Ca:P inicial debe ser $1: 1$ en Mmol o 1,3:1 en $\mathrm{mg}^{15}$.

Tabla 1 Resumen de publicaciones de hipofosfemia precoz en RNMBPN

\begin{tabular}{|c|c|c|c|c|c|c|}
\hline $\begin{array}{l}\text { Autor } \\
\text { Ichikawa, } \\
2012^{19}\end{array}$ & \multirow{2}{*}{$\frac{N}{58}$} & \multirow{2}{*}{$\begin{array}{l}\text { Criterios ingreso } \\
\text { RN }<1.000 \mathrm{~g} \\
\text { AEG y } P E G\end{array}$} & \multirow{2}{*}{\multicolumn{2}{|c|}{$\begin{array}{l}\text { Intervención } \\
\text { Aá día 1: } 0,5 \text { aumento } 0,5 \text { por día a } \\
2,5 \mathrm{~g} / \mathrm{k} / \mathrm{d} \\
\text { Ca: } \mathrm{P}=4-7: 1\end{array}$}} & \multicolumn{2}{|c|}{ Incidencia de hipofosfemia o valores observados } \\
\hline $\begin{array}{l}\text { Ichikawa, } \\
2012^{19}\end{array}$ & & & & & $\begin{array}{l}\text { Hipofosfemia }<2,7 \mathrm{mg} / \mathrm{dl} \text { día } \\
\text { PEG } \\
\text { AEG } \\
\text { Hipercalcemia > } 11 \mathrm{mg} / \mathrm{dl} \text { día } \\
\text { PEG } \\
\text { AEG }\end{array}$ & $\begin{array}{c}8 \\
53 \% \\
12 \% \\
8 \\
71 \% \\
56 \%\end{array}$ \\
\hline \multirow[t]{2}{*}{$\begin{array}{l}\text { Moltu, } \\
2012^{10}\end{array}$} & \multirow[t]{2}{*}{48} & \multirow[t]{2}{*}{$\mathrm{RN}<1.500 \mathrm{~g}$} & \multicolumn{2}{|c|}{$\begin{array}{l}\text { Aá sem 1: 3,7 g/k/d } \\
\text { P } 29 \mathrm{mg} / \mathrm{k} / \mathrm{d}\end{array}$} & $\begin{array}{l}\text { Fosfemia mínima sem } 1 \\
\text { Hipofosfemia }<4,2 \text { mg/dl } \\
\text { Sepsis }\end{array}$ & $\begin{array}{l}3,2 \mathrm{mg} / \mathrm{d} \\
77 \% \\
63 \%\end{array}$ \\
\hline & & & \multicolumn{2}{|c|}{$\begin{array}{l}\text { Aá sem 1: } 2,5 \mathrm{~g} / \mathrm{k} / \mathrm{d} \\
\text { P } 25 \mathrm{mg} / \mathrm{k} / \mathrm{d}\end{array}$} & $\begin{array}{l}\text { Fosfemia mínima sem } 1 \\
\text { Hipofosfemia }<4,2 \text { mg/dl } \\
\text { Sepsis }\end{array}$ & $\begin{array}{l}5,3 \mathrm{mg} / \mathrm{d} \\
26 \% \\
29 \%\end{array}$ \\
\hline \multirow[t]{3}{*}{$\begin{array}{l}\text { Bonsante, } \\
2013^{6}\end{array}$} & \multirow[t]{3}{*}{154} & \multirow[t]{3}{*}{$\mathrm{RN}<33$ sem } & \multicolumn{2}{|c|}{$\begin{array}{l}\text { Alpar día } 1, \text { Aá < 1,2 } \\
\text { g/k/d P } 16 \mathrm{mg} / \mathrm{k} / \mathrm{d}\end{array}$} & $\begin{array}{l}\text { Hipofosfemia severa }<3 \mathrm{mg} / \mathrm{dl} \\
\text { Con AA } 1,2 \mathrm{~g} / \mathrm{k} / \mathrm{d}\end{array}$ & $0 \%$ \\
\hline & & & \multicolumn{2}{|c|}{ Aá $1,8 \mathrm{~g} / \mathrm{k} / \mathrm{d}$ P $19 \mathrm{mg} / \mathrm{k} / \mathrm{d}$} & Con AA $1,8 \mathrm{~g} / \mathrm{k} / \mathrm{d}$ & $4,6 \%$ \\
\hline & & & \multicolumn{2}{|c|}{ Aá 2,3 g/k/d P $21 \mathrm{mg} / \mathrm{k} / \mathrm{d}$} & Con AA 2,3 g/k/d & $12,5 \%$ \\
\hline $\begin{array}{l}\text { Ross, } \\
2013^{18}\end{array}$ & 2.253 & $\begin{array}{l}\mathrm{RN}<1.500 \mathrm{~g} \\
\mathrm{AEG} \text { y } \mathrm{PEG}\end{array}$ & \multicolumn{2}{|c|}{$\begin{array}{l}\text { 2001-2005 Sol. glucosada, día 1-3 } \\
\text { 2005-2010 Sol. glucosada + Aá día } \\
\text { 1-3 }\end{array}$} & $\begin{array}{l}\text { PEG: hipofosfemia } \\
\text { AEG: hipofosfemia } \\
\text { Preclampsia OR } \\
\text { Período 2005-10 }\end{array}$ & $\begin{array}{r}41 \% \\
9 \% \\
2,58 \\
\text { OR } 3,13\end{array}$ \\
\hline $\begin{array}{l}\text { Christmann, } \\
2014^{14}\end{array}$ & 79 & $\mathrm{RN}<34 \mathrm{sem}$ & \multicolumn{2}{|c|}{$\begin{array}{l}\text { Ca } 100 \text { mg/dl } \\
\text { glicerofosfato } 48 \text { mg/dl }\end{array}$} & $\begin{array}{l}\text { Hipofosfemia < 5,5 mg/dl } \\
\text { Hipercalcemia }>10,6 \text { mg/dl }\end{array}$ & $\begin{array}{l}34 \% \\
45 \%\end{array}$ \\
\hline $\begin{array}{l}\text { Broubed, } \\
2015^{15}\end{array}$ & 48 & $\begin{array}{l}\text { RN 24-27 sem } \\
\text { AEG y PEG }\end{array}$ & \multicolumn{2}{|c|}{$\begin{array}{l}\text { Aá } 2 \text { g/k/d desde día } 1 \\
\text { Líp } 1 \mathrm{~g} / \mathrm{k} / \mathrm{d} \text { Gluc } 6 \mathrm{~g} / \mathrm{k} / \mathrm{d} \\
\text { Electrolitos después día } 1\end{array}$} & $\begin{array}{l}\text { Hipofosfemia }<4,8 \mathrm{mg} / \mathrm{dl} \\
\text { PEG } \\
\text { AEG }\end{array}$ & $\begin{array}{l}\text { 3-4 día } \\
40 \% \\
9 \%\end{array}$ \\
\hline $\begin{array}{l}\text { Brener, } \\
2015^{13}\end{array}$ & 61 & $\mathrm{RN}<1.250 \mathrm{~g}$ & \multicolumn{2}{|c|}{$\begin{array}{l}\text { Aá } 3-3,5 \mathrm{~g} / \mathrm{k} / \mathrm{d} \text { día } 1 \\
\text { Lípidos } 3 \mathrm{~g} / \mathrm{k} / \mathrm{d} \text { día } 1 \\
\text { Ca: P 2:1 día } 1\end{array}$} & $\begin{array}{l}\text { Hipofosfemia }<4 \text { mg/dl } \\
\text { Leve-moderada } 2-4 \mathrm{mg} / \mathrm{dl} \\
\text { Severa }<2 \mathrm{mg} / \mathrm{dl}\end{array}$ & $\begin{array}{l}91 \% \\
57 \% \\
34 \%\end{array}$ \\
\hline $\begin{array}{l}\text { Senterre, } \\
2015^{16}\end{array}$ & 102 & $\mathrm{RN}<1.250 \mathrm{~g}$ & \multicolumn{2}{|c|}{$\begin{array}{l}\text { Aá } 2,7 \mathrm{~g} / \mathrm{K} / \mathrm{d} \text { día } 1 \text { al } 3 \\
\text { Ca } 60 \text { y P } 40 \mathrm{mg} / \mathrm{k} / \mathrm{d}\end{array}$} & $\begin{array}{l}\text { Hipofosfemia }<5 \mathrm{mg} / \mathrm{dl} \\
\text { Severa }<3 \mathrm{mg} / \mathrm{dl} \\
\text { Hipercalcemia }\end{array}$ & $\begin{array}{r}37 \% \\
5 \% \\
13 \%\end{array}$ \\
\hline \multirow{3}{*}{$\begin{array}{l}\text { Moe, } \\
2015^{17}\end{array}$} & \multirow[t]{3}{*}{108} & \multirow{3}{*}{$\begin{array}{l}\mathrm{RN}<28 \text { sem } \\
\mathrm{AEG} \text { y } \mathrm{PEG}\end{array}$} & Aá 2,1 P 40 & $\mathrm{CaO}$ & Fosfemia promedio $2 \mathrm{sem}$ & $5,4 \pm 1,3$ \\
\hline & & & Aá 3,1 P 2 & Ca 20 & Fosfemia promedio 2 sem & $3 \pm 1,4$ \\
\hline & & & Aá $3,1 \quad P \quad 32$ & Ca 48 & Fosfemia promedio 2 sem & $4,2 \pm 1,1$ \\
\hline $\begin{array}{l}\text { Bustos, } \\
2017^{12}\end{array}$ & 20 & $\begin{array}{l}\mathrm{RN}<1.500 \mathrm{~g} \\
\text { Consentimiento } \\
\text { informado }\end{array}$ & $\begin{array}{l}\text { Aá } 2 \text { g/k día } 1 \\
\text { Fosforo día } 3\end{array}$ & io día 1 & $\begin{array}{l}\text { Hipofosfemia }<4 \text { mg/dl } \\
\text { Severa }<3 \text { mg/dl }\end{array}$ & $\begin{array}{l}45 \% \\
25 \%\end{array}$ \\
\hline $\begin{array}{l}\text { Mulla, } \\
2017^{15}\end{array}$ & 100 & $\begin{array}{l}\mathrm{RN}<37 \text { sem con } \\
\text { parenteral }\end{array}$ & $\begin{array}{l}\text { Ca: P 1,5:1-1, } \\
\text { Ca: P 1:1 mM }\end{array}$ & $\begin{array}{l}\text { iMol vs } \\
7 / 100 \mathrm{ml})\end{array}$ & $\begin{array}{l}\text { Hipofosfemia }<4,5 \mathrm{mg} / \mathrm{dl} \\
\text { Hipercalcemia }>12 \mathrm{mg} / \mathrm{dl}\end{array}$ & $\begin{array}{l}61 \text { vs } 35 \% \\
61 \text { vs } 20 \%\end{array}$ \\
\hline
\end{tabular}

RN recién nacido. AEG Adecuado para edad gestacional. PEG pequeño para edad gestacional. Aá aminoácidos. Lip lípidos. Gluc glucosa. Alpar alimentación parenteral. Sem semana. Sol solución. RNMBPN Recién nacido de muy bajo peso de nacimiento. Ca Calcio. P Fósforo. 
El aporte de fósforo incorpora sodio o potasio según la sal utilizada, los que pueden iniciarse con la nutrición parenteral el primer día. El balance de potasio es positivo en el $94 \%$ de los casos cuando el aporte de Aá es mayor de $2 \mathrm{~g} / \mathrm{k} /$ día y es negativo en el $92 \%$ cuando el aporte de AA es menor de $1,5 \mathrm{~g} / \mathrm{k} / \mathrm{d}^{26}$. El sodio puede ser iniciado en dosis de 1 a $1,8 \mathrm{mEq} / \mathrm{k}$ desde el primer día de vida, sin producir problemas ${ }^{8}$.

Programar adecuadamente el aporte parenteral y enteral y controlar fosfemia, tanto como se controla la calcemia en la primera semana, permitirá minimizar el riesgo. Dadas las limitaciones de volumen de aporte en los primeros días, probablemente es difícil un aporte $\geq 3 \mathrm{~g} / \mathrm{k} / \mathrm{d}$ de Aá con un aporte suficiente de calcio y fósforo. Iniciar la parenteral en forma más proporcionada, con Aá de alrededor de 2 g/k/día, con aumento progresivo, y con minerales y electrolitos proporcionales desde el primer día permitirá evitar hipofosfemia y reducir el impacto en la evolución respiratoria e infecciosa $^{8,15,17}$.

Una vez detectada la hipofosfemia puede ser necesario invertir la relación Ca:P a 0,6-0,8:1 en mg. en la solución parenteral hasta normalizar el fósforo, en especial, si no se cuenta con glicerofosfato de sodio, que contiene $1 \mathrm{mmol}$ de fósforo y $2 \mathrm{mEq}$ de sodio por $\mathrm{ml}$., y que es mucho más soluble con gluconato de calcio que el fosfato de potasio (tabla 2).

\section{Hipofosfemia tardía}

La hipofosfemia tardía también fue descrita en relación a desbalance del aporte de proteínas y de fósforo, pero hace más de 30 años, al cambiar del uso de leche de banco, a leche materna de su propia madre, con mayor contenido de proteínas, favorable para un mayor crecimiento, pero con igual cantidad de fósforo, muy bajo para cubrir requerimientos del MBPN. Nuevamente se observó hipofosfemia con hipercalcemia, hipercalciuria $^{27}$. El primer estudio de suplemento con sólo fósforo mostró que se debía administrar calcio y fósforo, lo que fue incorporado posteriormente a los fortificantes ${ }^{28}$. Los altos requerimientos de mineral en el prematuro son difíciles de alcanzar, especialmente cuando el aporte es parenteral y hay morbilidad y terapias que afectan la disponibilidad mineral. Así se produce la enfermedad metabólica ósea del prematuro (EMOP) con alteraciones que van desde una leve disminución de la mineralización ósea hasta la presencia de fracturas y/o signos radiológicos de raquitismo ${ }^{29,30}$. El contenido mineral óseo por DEXA (dual-energyxray-absorptiometry) es la medición más adecuada para evaluar la alteración, pero no se usa habitualmente en la clínica. La fosfemia baja asociada a aumento de las fosfatasas alcalinas es lo más empleado, actual-

\section{Tabla 2. Sugerencias de manejo de hipofosfemia precoz}

- Medir fosfemia de forma rutinaria en todos los RNPT extremos, con especial énfasis en aquellos con restricción del crecimiento intrauterino

- La medición debería ser realizada desde el $2^{\circ}$ día de vida, con el fin de documentar hipofosfemia si está presente y en base a esto definir conductas

- Dependiendo de los valores encontrados, realizar seguimiento metabólico y ajuste de aporte en parenteral

- Si es necesario, disminuir el calcio con el fin de dar mayor aporte de fosfatos

- Idealmente utilizar en las preparaciones parenterales glicerolfosfato, que permite aumentar el fósforo sin mayor riesgo de precipitación en la solución de nutrición parenteral

mente los niveles altos de PTH con fosfemia disminuida parecen ser los mejores marcadores clínicos ${ }^{31}$. Generalmente los niveles de $25 \mathrm{OH}$ vitamina D están normales o levemente reducidos y la PTH elevada por hiperparatiroidismo secundario. La calcemia es normal y el fósforo es bajo. En caso de calcemia baja debe plantearse déficit de vitamina $\mathrm{D}$, que puede ser por aporte o por alteración congénita de hidroxilación ${ }^{32}$. Para el seguimiento clínico la excreción urinaria de Ca y P entre 1 y $2 \mathrm{mmol} / \mathrm{L}$ en muestra aislada permite individualizar los requerimientos de los minerales. El aporte óptimo produce una excreción urinaria de Ca menor a $4,8 \mathrm{mg} / \mathrm{dL}(1,2 \mathrm{mmol} / \mathrm{L})$ y de $\mathrm{P}$ mayor a $1,2 \mathrm{mg} / \mathrm{dL}(0,4 \mathrm{mmol} / \mathrm{L})^{30}$.

Factores de riesgo de la enfermedad metabólica ósea son: nutrición parenteral prolongada, retraso en el aporte enteral, displasia broncopulmonar, enterocolitis necrotizante, uso de diuréticos, de prostaglandinas, de metilxantinas, de corticoides, muy bajo peso de nacimiento y RCIU ${ }^{30}$.

A nivel óseo se presentan signos de raquitismo con ensanchamiento epifisiario y deshilachamiento, y eventualmente fracturas patológicas. Este cuadro se ve agravado con uso de diuréticos que pueden aumentar la hipercalciuria y producir nefrocalcinosis. La identificación sistemática de las radiografías en un centro neonatal inglés muestra una incidencia de fracturas costales del $2 \%{ }^{32}$. El compromiso de la densidad ósea puede afectar la masa ósea en la adultez y definir mayor riesgo de patología ósea en la edad adulta. Existe evidencia de que un programa regular de movimientos pasivos en el prematuro favorece una mineralización más adecuada ${ }^{33}$.

El control debe iniciarse desde las 4 semanas, a los prematuros en riesgo: MBPN o con tratamiento con diuréticos o corticoides: con medición de calcemia, fosfemia, y fosfatasas alcalinas (si se puede niveles séricos de $\mathrm{PTH}$ ) semanal o quincenal, según riesgo y valores. 
Hipofosfemia en recién nacidos - M. P. Cubillos C. et al

\section{Tabla 3 Tamizaje y manejo de hipofosfemia tardía}

- Recién nacidos prematuros extremos o muy bajo peso de nacimiento

- Prematuros con uso de diuréticos o corticoides prolongado

Control desde las 4 semanas con calcio, fósforo, fosfatasas alcalinas y si se puede PTH

- $\quad$ Si fósforo $<5 \mathrm{mg} / \mathrm{dl}$ suplementar con calcio y fósforo adicional

- Vitamina D hasta 1.000 UI diarias

- Si persiste aumento de fosfatasas alcalinas controlar calciuria y fosfaturia

Si fosfemia menor de $5 \mathrm{mg} / \mathrm{dl}$ suplementar calcio y fósforo adicional. Si fosfatasas alcalinas continúan aumentando: Controlar aporte con calciuria y fosfaturia. Se debe asegurar un óptimo aporte de Vitamina D: hasta 1.000 UI diaria, considerando aporte enteral total y parenteral, controlando niveles si es posible. Minimizar el uso de diuréticos o corticoides reducirá el riesgo de enfermedad metabólica ósea.

A largo plazo, la hipofosfemia en el período neonatal, se ha asociado a alteraciones del esmalte dentario, como único factor identificado entre los factores perinatales evaluados ${ }^{34}$. También se ha asociado a un muy discreto menor crecimiento a los dos años, controlando por grado de prematurez y de $\mathrm{RCIU}^{35}$ (tabla 3 ).

\section{Conclusiones}

La revisión de este tema tiene como fin concientizar a los clínicos que trabajan con recién nacidos prematuros, acerca de la importancia de la nutrición como parte del manejo integral, intensivo y precoz. El revisar nuestras prácticas clínicas es esencial para mejorar la calidad de atención de los pacientes. Hoy en día, existen alteraciones metabólicas asociadas a las nuevas recomendaciones nutricionales que inician aminoácidos de forma precoz y en alta cantidad, por lo que es importante hacer el seguimiento de minerales especialmente durante los primeros días de vida. La aparición de hipofosfemia precoz será detectada, si existe, en los controles iniciales y permitirá a los clínicos hacer las correcciones en las preparaciones de parenterales con el fin de optimizar el aporte necesario de nutrientes en los recién nacidos prematuros.

\section{Conflicto de intereses}

Los autores declaran no tener conflicto de intereses.

\section{Referencias}

1. Kovacs C. Maternal mineral and bone metabolism during pregnancy,lactation, and post-weaning recovery. Physiol Rev. 2016;96:449-547.

2. Linglart A, Chaussain C. Endocrine control of serum phosphate: from thediscoveries of phosphatonins to novel therapies. Ann Endocrinol (Paris). 2016;77Suppl 1:S36-S42.

3. Husain SM, Mughal MZ, Tsang R. Calcium, Phosphorus and Magnesium transport across placenta. En Richard A. Polin, MD. Fetal and Neonatal physiology. Fourth Edition 2011;364-73.

4. Kovacs CS. Calcium, phosphorus, and bone metabolism in the fetus and newborn Early Hum Develop. 2015;91:623-8

5. Mimouni F, Mandela D, Lubetzky R, Senterren R. Calcium, Phosphorus, Magnesium and Vitamin D Requeriments of the preterm infant. Koletzko B, Poindexter B, Uauy R (eds): Nutritional Care of Preterm Infants: Scientific Basis and Practical Guidelines.World Rev Nutr Diet. Basel, Karger. 2014; 110:140-51.

6. Bonsante F, Iacobelli S, Latorre G, et al. Initial Amino Acid Intake Influences Phosphorus and CalciumHomeostasis in Preterm Infants-It Is Time to Change the Composition ofthe Early Parenteral Nutrition. www.plosone.org August 2013; Volume 8:Issue 8.

7. Thureen PJ, Melara D, Fennessey PV, Hay WW Jr. Effect of low versus high intravenous amino acid intake on very low birth weight infants in the early neonatal period. Pediatr Res. 2003;53:2432.

8. Senterre T, Rigo J. Optimizing Early Nutritional Support Based on Recent Recommendations in VLBW Infants and Postnatal Growth Restriction. J Pediatr Gastroenterol Nutr. 2011;53:536-42.

9. Stephens BE, Walden RV, Gargus RA, et al. First-week protein and energy intakes are associated with 18-month developmental outcomes in extremely low birth weight infants. Pediatrics. 2009;123:1337-43.

10. Moltu S, Strommen K, Blakstad E, et al. Enhanced feeding in very-low-birthweight infants may cause electrolyte disturbances and septicemia e A randomized, controlled trial. Clinical Nutrition. 2013;32: 207-12 .

11. Boubred F, Herlenius E, Bartocci M, Jonsson B, Vanpee M. Extremely preterm infants who are small for gestational age have a high risk of early hypophosphatemia and hypokalemia. ActaPædiatrica 2015;104:1077-83.
12. Bustos Lozano G, Hidalgo Romero Á, Melgar Bonis A, Ureta Velasco N, Orbea Gallardo C, Pallás Alonso C. Early hypophosphataemia in at risk newborns. Frequency and magnitude. An Pediatr (Barc). 2017 Jun 3. pii: S16954033(17)30186-8.

13. Brener P, Galletti MF, Fernández J onusas S, Alonso G, Mariani G, Fustiñana C. Early hypophosphatemia in preterm infants receiving aggressive parenteral nutrition. J Perinatol. 2015;35:712-5.

14. Christmann V, De Grauw A, Visser R, Matthijsse R, Van Goudoever J, VanHeijst A. Early Postnatal Calcium and Phosphorus Metabolism in Preterm Infants. J Pediatr Gastroenterol Nutr. 2014;58:398-403.

15. Mulla S, Stirling S, Cowey S, et al. Severe hypercalcaemia and hypophosphataemia with an optimised preterm parenteral nutrition formulation in two epochs of differing phosphate supplementation. ArchDisChild Fetal Neonatal Ed. 2017;102:F451-F455.

16. Moe K, Beck-Nielsen S, Lando A, Greisen G, Zachariassen G. Administering different levels of parenteral phosphate and amino acids did not influence growth in extremely preterm infants. Acta Paediatr. 2015; 104:894-9. 
17. Senterre T, Zahirah I, Pieltain C, Halleux V, Rigo J. Electrolyte and mineral homeostasis after optimizing early macronutrient intakes in VLBW infantson parenteral nutrition. J Pediatr Gastroenterol Nutr. 2015;61:491-8.

18. Ross J, Finch C, Taylor S. Refeeding syndrome in very-low-birthweightintrauterine growth-restricted neonates. J Perinatol. 2013; 33:717-20.

19. Ichikawa G, Watabe $Y$, Suzumura $\mathrm{H}$, Sairenchi T, Muto T, Arisaka O. Hypophosphatemia in small for gestational age extremely low birth weight infants receiving parenteral nutrition in the first week after birth. J Pediatr Endocr Met. 2012;25:317-3.

20. Mena P, Cubillos MP, Toro C, Zúñiga C. Perfil bioquímico en sangre de cordón en prematuros extremos y crecimiento fetal. Rev Chil Pediatr. 2016;87:250-4.

21. Crook M. Refeeding syndrome: Problems with definition and management. Nutrition. 2014;30:1448-55.

22. Mizumoto H, Mikami M, Oda H, Hata D. Refeeding syndrome in a small-fordatesmicro-preemie receiving early parenteral nutrition. Pediatr Int. 2012;54:715-7.

23. Marinella MA. The refeeding syndrome and hypophosphatemia. Nutr Rev. 2003;61:320-3.

24. Mize C, Corbett R, Uauy R, Nunnally R, Williamson S. Hypotonia of Rickets: A Sequential Study by P-31 Magnetic Resonance Spectroscopy. Pediatr Res. 1988; 6:713-6.

25. Dreyfus L, Fischer C, Remontet L, et al. Low phosphatemia in extremely low birth weight neonates: A risk factor for hyperglycemia? Clin Nutr. 2016;35:105965.

26. Guellec I, Gascoin G, Beuchee A, et al. Biological Impact of Recent Guidelines on ParenteralNutrition in Preterm Infants. J Pediatr Gastroenterol Nutr. 2015; 61: 605609.

27. Lyon AJ, McIntosh N. Calcium and phosphorus balance in extremely lowbirthweight infants in the first six weeks of life. Arch Dis Child. 1984; 59:1145-50.

28. Senterre J, Putet G, Salle B, Rigo J. Effects of vitamin $D$ and phosphorus supplementation on calcium retention in preterm infants fed banked human milk. J Pediatr. 1983;103:305-7.

29. Pieltain C, de Halleux V, SenterreTh, et al. Prematurity and Bone Health. World Rev Nutr Diet. 2013; 106:181-8.
30. Harrison CM, Gibson AT. Osteopenia in preterm infants. Arch Dis Child Fetal Neonatal Ed. 2013;98:F272-5.

31. Moreira A, Swischuk L, Malloy M, Mudd D, Blanco C, Geary C. Parathyroid hormone as a marker for metabolic bone disease of prematurity J Perinatol2014; 34: 787-91.

32. Lucas-Herald A, Butler S, Mactier H, McDevitt H, Young D, Ahmed SF. Prevalence and characteristics of rib fractures in ex-preterm infants. Pediatrics. 2012;130:1116-9.

33. Moyer-Mileur LJ, Brunstetter V, Mc Naught TP, Gill G, Chan GM. Daily physical activity program increases bone mineralization and growth in preterm very low birth weight infants. Pediatrics. 2000;106:1088-92.

34. Merheb R, Arumugam C, Lee W, Collin M, Nguyen C, Groh-Wargo S, Nelson S.Neonatal Serum Phosphorus Levels and Enamel Defects in Very Low Birth Weight Infants. J Parenter Enteral Nutr. 2016;40:835-41.

35. Isojima T, Kushima R, Goishi K, Tsuchida S, Watanabe T, Takahashi N, KitanakaS. Mineral status of premature infants in early life and linear growth at age 3 . Pediatr Int. 2015;57:864-9. 